\title{
Genome-wide association studies on prostate cancer: the end or the beginning?
}

\author{
Rui Chen ${ }^{*}$, Shancheng Ren ${ }^{*}$, Yinghao Sun ${ }^{\bowtie}$ \\ Department of Urology, Shanghai Changhai Hospital, Second Military Medical University, Shanghai 200433, China \\ $\square$ Correspondence: sunyh@medmail.com.cn \\ Received July 10, 2013 Accepted July 31, 2013
}

\begin{abstract}
Prostate cancer ( $\mathrm{PCa})$ is the second most frequently diagnosed malignancy in men. Genome-wide association studies (GWAS) has been highly successful in discovering susceptibility loci for prostate cancer. Currently, more than twenty GWAS have identified more than fifty common variants associated with susceptibility with $\mathrm{PCa}$. Yet with the increase in loci, voices from the scientific society are calling for more. In this review, we summarize current findings, discuss the common problems troubling current studies and shed light upon possible breakthroughs in the future. GWAS is the beginning of something wonderful. Although we are quite near the end of the beginning, post-GWAS studies are just taking off and future studies are needed extensively. It is believed that in the future GWAS information will be helpful to build a comprehensive system intergraded with PCa prevention, diagnosis, molecular classification, personalized therapy.
\end{abstract}

KEYWORDS prostate cancer, genome-wide association study

\section{INTRODUCTION}

\section{Prostate cancer}

Prostate cancer ( $\mathrm{PCa}$ ) is the second most frequently diagnosed malignancy and the sixth leading cause of cancer-related death in men, with an estimated 914,000 newly diagnosed cases and 258,000 deaths per year globally (Siegel et al., 2013). Among all cancers, prostate cancer is one of the most heritable with genetic factors estimated to account for $42 \%$ of the risk (Lichtenstein et al., 2000). The genetic background of PCa has been extensively studied, but the known genetic factors only have a moderate influence on PCa susceptibility.

\footnotetext{
*These authors contributed equally to the work.
}

The difficulty in PCa research mostly lies in early diagnosis and precise therapy. Prostate-specific antigen (PSA) screening has been the standard care on detecting PCa but this biomarker has been characterized to have low specificity, causing over-diagnosis and over-treatment. In spite of the discovery of new biomarkers, the result of early diagnosis of $\mathrm{PCa}$ is still unsatisfactory. Secondly, as evidence began to accumulate that $\mathrm{PCa}$ is a heterogenic disease, the genetic background of $\mathrm{PCa}$ is different in many ways. Berger $\mathrm{M}$ and coworkers (Berger et al., 2011) illustrated that in different patients, the alterations and rearrangements are different using whole genome genotyping techniques. Results of other institute (Mehra et al., 2007) confirmed that multifocal PCa is also a heterogeneous group of diseases caused by different genetic changes and manifested distinctive behaviors. Such vague in susceptibility and oncogenesis of PCa may help to explain the dilemma we met in dealing with clinical therapy: we targeted different tumors with the same strategy, revealing this molecular heterogeneity is critical to the future development and utility of diagnostic and prognostic biomarkers. Such fact calls for comprehensive solution to a better molecular classification of PCa through genetic perspective.

\section{Genome-wide association studies}

Genome-wide association studies (GWAS) have been highly successful in discovering susceptibility loci for prostate cancer and it has rapidly become a standard weapon in the arsenal of investigators interested in the genetic origin of prostate cancer. Indeed, since the first prostate cancer GWAS in 2005, more than 1300 studies have been added to the Catalog of Published Genome-Wide Association Studies (Juran and Lazaridis, 2011). Before the application of GWAS techniques, genome-wide linkage analysis is the most powerful tool to detect disease genetic background. However, as most common diseases are determined by the sum of multiple genetic and 
environmental factors and the linkage study has limited power for identifying common genetic variants that have modest effects on disease (Hirschhorn and Daly, 2005).

The standardized GWAS are usually carried out in multistage fashion. DNA from hundreds or thousands of patients and controls will be genotyped using commercially available microarray chips. Even if the price of genotyping has been substantially decreased in the past few years, the total cost for genotyping a large sample size using more single-nucleotide polymorphisms (SNPs) can be huge. However, the multistage designs can retain most of the power of the design with about half of the cost (Elston et al., 2007). In a general situation, the whole study consists of two or three phases. In the first phase, a full set of SNPs across the genome, the genotypes of patients and controls are compared to identify SNPs possibly associated with cancer risk. Only $5 \%-10 \%$ most significant SNPs will be carried out into the next phase in a much larger set of independent patients and controls. In the third phase, the SNPs found previously will be tested in a larger and more heterogeneous patient and control groups (Stadler et al., 2010). One of the key to successful GWAS is to repeat the test to yield more significant association. The GWAS is all about replication, replication and replication.

\section{CURRENT GWAS IN PROSTATE CANCER}

\section{The history of GWAS in prostate cancer}

The first GWAS in prostate cancer (PCa) was carried out in 2007, revealing the second risk locus at $8 q 24$, rs6983267, to be associated with PCa (Yeager et al., 2007). Ever since then, more than twenty GWAS have identified more than 50 common variants associated with susceptibility with $\mathrm{PCa}$, which is quite high compared to the corresponding numbers for other types of cancers and other common diseases.

These GWAS are carried out to investigate the association of genetic changes with PCa as well as other aspect in therapy such as erectile dysfunction and prostate cancer treatment, gene-gene interaction (For a list of GWAS, see http://www. genome.gov/26525384). Here, we summarize the findings of GWAS and possible functional explanation of some SNPs that have been extensively reported (Table 1). As is shown in Table 1, most of these SNPs are located in intergenic regions and only some of these genes have been given possible functional explanations.

\section{$8 q 24$}

Chromosome 8q24 region was first identified via the genomewide linkage analysis in Icelandic families with prostate cancer. Since the first GWAS in PCa indicated the second locus on $8 q 24$ is correlated with PCa susceptibility, such association was confirmed by other GWAS and replication studies (Gudmundsson et al., 2007, 2008; Eeles et al., 2008; Thomas et al., 2008; Yeager et al., 2009; Takata et al., 2010; Schumacher et al., 2011; Cheng et al., 2012). Several SNPs, rs10090154, rs1016343, rs1447295, rs16901979, rs4242382, rs445114, rs6470494, rs6983267, rs6983561, and rs7000448, have been reported to have a significant association with $\mathrm{PCa}$. Among them, rs1447295 and rs6983267 are the most frequently reported SNP on 8q24 (Gudmundsson et al., 2007, 2008; Eeles et al., 2008; Thomas et al., 2008; Yeager et al., 2009; Schumacher et al., 2011). Mapping results indicated that it is on the intron region of LOC727677. The function of LOC727677 is uncharacterized. Rs6983267 is $70 \mathrm{~kb}$ centromeric to rs1447295, but they are not linkage disequilibrium (LD) (Yeager et al., 2007). The 8q24 region has been previously considered as a $600-k b p$ region without genes with little or no transcriptional activity. However, nowadays there is evidence that suggest possible mechanisms. POU5F1P1, originally thought to be a pseudogene, has been identified on $8 q 24$. It is now believed that POU5F1P1 can encode a functional protein that contributes to carcinogenesis through its role as a weak transcriptional activator (Panagopoulos et al., 2008). Additionally, recent studies have shown that 8q24 encodes enhancers of the nearby oncogene MYC (Sotelo et al., 2010; Wasserman et al., 2010). Wasserman et al. (2010) and Sotelo et al. (2010) both identified an enhancer of MYC that contained rs6983267. In a follow-up study of the Cancer Genetic Markers of Susceptibility (CGEMS) study, 8q24 rs4242382 and rs6983267 were proved to be associated with risk for metastatic prostate cancer (Ahn et al., 2011).

\section{MSMB}

Four GWAS (Eeles et al., 2008; Thomas et al., 2008; Takata et al., 2010; Schumacher et al., 2011) identified $\beta$-microseminoprotein (MSMB) promoter SNP, rs10993994, to be associated with PCa risk. PSP94 is the protein MSMB coded. Further studies demonstrate that the variant allele affects expression of PSP94 and also suggest that it affects mRNA expression levels of an adjacent gene named NCOA4 (FitzGerald et al., 2013). The gene product of NCOA4 interacts directly with the androgen receptor as a co-activator to enhance AR transcriptional activity. It is recently reported that the expression of full-length MSMB-NCOA4 fusion transcripts is regulated by the MSMB promoter (Lou et al., 2012). Computational network analysis reveals that the MSMB gene is functionally connected to NCOA4 and the androgen receptor signaling pathway (Lou et al., 2012). Another functional study (Chang et al., 2009) proved the prostate cancer risk associated T allele of rs10993994 had a much lower promoter activity compared with the $\mathrm{C}$ allele. Treatment with increasing concentrations of the synthetic androgen (R1881) resulted in a dose-dependent increase in promoter activity of the $\mathrm{C}$ allele, but not the $\mathrm{T}$ allele. Further, this allele has also been illustrated to be associated with risk for metastatic prostate cancer in a follow-up study in the Cancer Genetic Markers of Susceptibility (CGEMS) database (per variant allele summary $\mathrm{RR}=1.24,95 \% \mathrm{Cl}=$ 1.05-1.48) (Ahn et al., 2011). 
Table 1. Extensively reported susceptibility loci in GWAS of PCa

\begin{tabular}{|c|c|c|c|c|c|}
\hline Region & Reported gene(s) & Mapped gene(s) & SNPs & Context & Ref \\
\hline \multirow[t]{9}{*}{$8 q 24$} & Intergenic & LOC727677 & rs1447295 & intron & $\begin{array}{l}\text { Gudmundsson et al., 2007; Gudmundsson et } \\
\text { al., 2008; Yeager et al., } 2007\end{array}$ \\
\hline & Intergenic & SRRM1P1-POU5F1B & rs6983267 & intergenic & $\begin{array}{l}\text { Eeles et al., 2008; Thomas et al., 2008; } \\
\text { Yeager et al., 2009; Schumacher et al., } 2011\end{array}$ \\
\hline & Intergenic & SRRM1P1-POU5F1B & rs445114 & intergenic & $\begin{array}{l}\text { Gudmundsson et al., 2008; Schumacher et } \\
\text { al., } 2011\end{array}$ \\
\hline & Intergenic & SRRM1P1-POU5F1B & rs6983561 & intergenic & Cheng et al., 2012 \\
\hline & Intergenic & SRRM1P1-POU5F1B & rs1456315 & intergenic & Takata et al., 2010; Xu et al., 2012 \\
\hline & Intergenic & SRRM1P1-POU5F1B & rs16901979 & intergenic & $\begin{array}{l}\text { Gudmundsson et al., 2007; Gudmundsson et } \\
\text { al., } 2008\end{array}$ \\
\hline & NR & POU5F1B-MYC & rs10090154 & intergenic & Cheng et al., 2012 \\
\hline & Intergenic & POU5F1B-MYC & rs4242382 & intergenic & Thomas et al., 2008 \\
\hline & Intergenic & PCAT1-SRRM1P1 & rs1016343 & intergenic & Eeles et al., 2008; Schumacher et al., 2011 \\
\hline $17 q 24.3$ & Intergenic & CALM2P1-SOX9 & rs1859962 & intergenic & $\begin{array}{l}\text { Gudmundsson et al., 2007; Gudmundsson et } \\
\text { al., 2008; Eeles et al., 2009; Schumacher et } \\
\text { al., } 2011\end{array}$ \\
\hline \multirow[t]{4}{*}{$11 q 13$} & Intergenic & MIR3164-MYEOV & rs7931342 & intergenic & Eeles et al., 2008 \\
\hline & Intergenic & MIR3164-MYEOV & rs7130881 & intergenic & Eeles et al., 2009; Schumacher et al., 2011 \\
\hline & Intergenic & MIR3164-MYEOV & rs10896449 & intergenic & Thomas et al., 2008 \\
\hline & Intergenic & MIR3164-MYEOV & rs11228565 & intergenic & Gudmundsson et al., 2008 \\
\hline $10 q 11.23$ & MSMB & MSMB & rs10993994 & nearGene-5 & $\begin{array}{l}\text { Eeles et al., 2008; Thomas et al., 2008; } \\
\text { Takata et al., 2010; Schumacher et al., } 2011\end{array}$ \\
\hline $19 q 13.33$ & KLK3 & $\mathrm{KLK} 2 / 3$ & rs2735839 & intergenic & Eeles et al., 2008 \\
\hline $7 \mathrm{p} 15.2$ & JAZF1 & JAZF1 & rs10486567 & intron & Thomas et al., 2008 \\
\hline \multirow[t]{2}{*}{ Xp11.22 } & NUDT10, NUDT11 & $\begin{array}{l}\text { NUDT10, NUDT11, } \\
\text { LOC340602 }\end{array}$ & rs5945572 & intergenic & Gudmundsson et al., 2008 \\
\hline & NUDT10, NUDT11 & NUDT11-CENPVP3 & rs5945619 & intergenic & Eeles et al., 2008 \\
\hline \multirow[t]{2}{*}{$17 q 12$} & Intergenic & HNF1B & rs4430796 & intron & $\begin{array}{l}\text { Gudmundsson et al., 2007; Gudmundsson et } \\
\text { al., 2008; Thomas et al., } 2008\end{array}$ \\
\hline & TCF2 & HNF1B & rs7501939 & intron & $\begin{array}{l}\text { Eeles et al., 2008; Eeles et al., 2009; Takata } \\
\text { et al., 2010; Schumacher et al., } 2011\end{array}$ \\
\hline $19 q 13.4$ & LILRA3 & MIR4752-LILRA3 & rs103294 & intergenic & Xu et al., 2012 \\
\hline
\end{tabular}

\section{$K L K 2-3$}

SNP rs2735839 is found to be associated with $\mathrm{PCa}\left(P=1 \times 10^{-18}\right)$ in a GWAS in 2008 (Eeles et al., 2008) and further confirmed in replication studies (Hooker et al., 2010; Bensen et al., 2013). This SNP has been suggested to have specific function as it lies between the KLK2 and KLK3 genes which have been reported to influence PCa risk (Severi et al., 2006). KLK3 encodes PSA protein (Ahn et al., 2008; Eeles et al., 2008). PSA has been widely used as a screening and diagnostic biomarker in prostate cancer and multiple SNPs in the promoter region of KLK3 have been associated with PSA concentrations and prostate cancer risk (Lai et al., 2007). While KLK2 encodes kallikrein-related peptidase 2 (hK2) which has also been reported to have the ability to reduce unnecessary biopsy in previously unscreened men with elevated total PSA (Vickers et al., 2010).

\section{HNF1B}

SNPs rs4430796 and rs7501939 on 17q12 are reported in GWAS (Gudmundsson et al., 2007, 2008; Eeles et al., 2008; Thomas et al., 2008; Eeles et al., 2009; Takata et al., 2010; Schumacher et al., 2011) and replication studies (Stevens et al., 2010; Kim et al., 2011) to be associated with PCa risk. These two SNPs are located in the first and second intron of the HNF1B (a transcription factor, TCF2) gene, respectively. Fine mapping results indicate that ten SNPs on HNF1B were significantly related to prostate cancer risk at a genome-wide significance level of $P<5 \times 10^{-8}$ with the most significant association with rs4430796 $\left(P=1.62 \times 10^{-24}\right)$. SNPs in HNF1B are associated with an increased risk of diabetes and a decreased risk of prostate cancer. Therefore, the possibility that the SNP-prostate cancer relationship is mediated by diabetes requires consideration. The association of the HNF1B SNPs 
with PCa should be substantially reduced or eliminated when take diabetic status into consideration.

\section{JAZF1}

SNP rs10486567 on JAZF1 (juxtaposed with another zinc finger 1) was reported to be associated with PCa susceptibility (Thomas et al., 2008). This SNP is located within intron 2 of JAZF1 gene on chromosome $7 \mathrm{p} 15.2$ and it has been showed a promising association with $\mathrm{PCa}$ overall $(P=2.14$ $\left.\times 10^{-6}\right)$ and a stronger association with aggressive $\mathrm{PCa}$ $\left(P=1.2 \times 10^{-7}\right)$. Follow-up replication studies have confirmed its association with $\mathrm{PCa}$ but not with aggressive $\mathrm{PCa}$ in European and African descent (Stevens et al., 2010; Chang et al., 2011). Researchers undertook fine mapping at $7 q 15.2$ to find rs10486567 is likely to be responsible locus (Prokunina-Olsson et al., 2010). GWAS data show that JAZF1 variation is also associated with type 2 diabetes (T2D) (Zeggini et al., 2008). A meta-analysis of GWAS data shows that T2D patients have a lower risk to develop PCa (Kerns et al., 2013). Since this SNP lies in intron 2 of JAZF1 and is not known to alter any apparent splicing or expression of this gene, the functional significance of this association has yet to be determined. Since JAZF1 variants were also shown to be associated with $\mathrm{T} 2 \mathrm{D}$, human height, suggesting the role it played in regulation of growth and metabolism. These findings suggest that JAZF1 and HNF1B influence $\mathrm{PCa}$ risk in metabolism regulation and $\mathrm{PCa}$ may have potential association with metabolism syndrome.

\section{LILRA3}

In our recent study, we identified the rs103294 on 19q13.4 to be associated with prostate cancer risk in Han Chinese $\left(P=5.34 \times 10^{-16}\right)($ Xu et al., 2012). The rs103294 marker at $19 q 13.4$ is in strong linkage equilibrium with a $6.7-k b$ germline deletion that removes the first six of seven exons in leukocyte immunoglobulin-like receptor A3 (LILRA3). LILRA3 is a gene regulating inflammatory response, and was significantly associated with the mRNA expression of LILRA3 in T cells $(P<$ $\left.1 \times 10^{-4}\right)$. LILRA3 has previously been reported to be involved in psoriatic patients and multiple sclerosis nevertheless it has never been reported to be associated with cancer risk. Since inflammation is involved in cancer pathogenesis, further studies may pay more attention to the role of LILRA3 played in other kinds of tumor.

There are much more SNPs that have been found and investigated than we have discussed here. For more information about these SNPs, it would be much more helpful to undergo those GWAS papers and following studies rather than reading this review. Our mission is just to illuminate the entrance so that those who are interested in these SNPs can go depth.

\section{Racial differences in PCa susceptibility SNPs}

According to the common disease-common variant hypothesis (Hirschhorn and Daly, 2005), common diseases such as prostate cancer is likely to be the consequence of common genetic variants with modest effect. Since the genetic variants are common, they are likely to be found in different populations of distinctive ancestors (Seng and Seng, 2008). The replication studies in other population have validated this hypothesis when they found that most of the published SNPs found in European population are repeatable in men of African and Asian ancestor (Chang et al., 2011; Ishak and Giri, 2011; Chan et al., 2012). Nevertheless, some studies indicated some of these SNPs are different in different population.

In the population group with the highest incidence rate, African Americans, some of the existing susceptibility loci such as SNP at JAZF1, MSMB, 11q13, and NUDT10/11, are also confirmed in different studies (Hooker et al., 2010; Chang et al., 2011); however, some loci failed to have significant difference in the replication studies. These unconfirmed loci may not really be loci that are not biological insignificant because these replication studies may have a lower statistic power. Interestingly, Haiman and colleagues (2011) demonstrated that many loci on chromosome 8q24 are more common in Africans than Europeans. The markers found to be associated with risk at each locus improved risk modeling in African Americans over the alleles reported in the original GWAS. This result possibly suggests a partial explanation for the higher incidence of prostate cancer in men of African descent and at the same time give us a hint about the important role that $8 q 24$ may play in PCa pathogenesis.

Different loci have been found in the Asian population. Take the Japanese GWAS as an example, researchers have found five SNPs to be associated with PCa susceptibility: $5 p 15$, GPRC6A/RFX6, 13q22, C2orf43, and FOXP4 (Takata et al., 2010). For the 31 previously reported loci in European populations, researchers confirmed the association of nine SNPs at $P<1.0 \times 10^{-7}$ and ten SNPs at $P<0.05$ in the Japanese population. Further study in Chinese has confirmed three of the newly discovered five SNPs to be associated with PCa (Wang et al., 2012). In the Chinese GWAS in prostate cancer, we reported two new loci on chromosomes 9q31.2 (rs817826, $P=$ $\left.5.45 \times 10^{-14}\right)$ and 19q13.4 (rs103294, $\left.P=5.34 \times 10^{-16}\right)$. Since GWAS in European population has been extensively carried out, the absence of three loci may indicate a possible hypothesis that these changes are more common in the Asian population. These findings may help us understand the pathogenesis of PCa in different racial groups and may be associated with the discrepancy in disease incidence. All in all, the replications in other ancestor descent confirmed the associations at some prostate cancer susceptibility loci originally identified in European descent populations and GWAS in other population have identified novel loci that are associated with PCa in Asian populations. Despite the similarity in susceptibility SNPs, there are some differences among different races in heterogeneity of genetic etiology or in the pattern of genetic variation.

\section{SNPs with aggressiveness of $\mathrm{PCa}$}

Since PCa is a heterogeneous disease, the prognosis outcome between indolent and aggressive cancer patients is 
vastly different. It is predicted that about $80 \%$ male may have latent PCa at the age of 80 (Konety et al., 2005). It is not clear whether these discovered susceptibility SNPs are associated with disease aggressiveness. Three SNPs on 10q26 (rs10788165, rs10749408, and rs10788165) and two SNPs on $15 q 21$ (rs4775302 and rs1994198) were found to be associated with aggressive PCa (Nam et al., 2011). Researchers further validated these SNPs in a biopsy cohort to find that the combinations of these five SNPs can predict intermediate to high-risk disease from low-risk cancer patients with odds ratio up to three. FitzGerald and colleagues (2011) performed genome-wide scan in aggressive PCa patients to successively identified rs6497287, a variant on $15 q 13$, to be associated with more aggressive than less aggressive disease. Recent metaanalysis of GWAS showed that rs 4054823 is associated with aggressive disease, but there is no annotated gene around this SNP and no explanation has been illustrated about its biological association with PCa aggressiveness (Xu et al., 2010). Rs11672691 at $19 q 13$ is significantly associated with aggressive PCa but not indolent PCa (Amin Al Olama et al., 2013). Most of these discovered susceptibility loci have been shown to be associated with aggressive PCa so far and further studies should pay more attention to aggressive type of PCa since they are more clinically significant.

\section{Annotation and functional study of susceptibility loci}

The real challenge for GWAS research in prostate cancer lies in the functional studies which will translate genetic data into biological meanings and thereafter into clinical benefits. However, annotation and functional studies of susceptibility loci are "naturally" difficult because that GWAS approach is based on the principle of linkage disequilibrium (LD); therefore, the genetic markers identified are unlikely to be the disease variants (Seng and Seng, 2008). Extensive re-sequencing and fine mapping are required to discern the disease variants. It is a great challenge in fine mapping when the SNPs within the genomic region are in strong LD. These are two sides of a coin, on one hand strong LD helps to reduce the number of markers to genotype in the genome-wide scan, on the other hand strong LD would limit the ability to "resolve" the association and creates difficulty to identify the "culprits".

After all, proposals have been raised to deal with the difficulties bothered the whole GWAS community. One thing that should be kept deeply in mind is that we must resist the temptation to equate any partial functional evidence as sufficient. Some pioneer investigators share their understanding of principles in post-GWAS functional studies (Freedman et al., 2011) which may be helpful guiding instructions.

\section{COMMON PROBLEMS OF CURRENT GWAS}

After more than 1000 GWAS published, researchers around the world can't help to ask: what can these costing researches bring us? Did GWAS help us to find better diagnosis biomarkers? Did GWAS help us to have a better treatment? The an- swer is still "not yet". So, the initial enthusiasm for GWASs is decreasing, and the GWAS society is facing severe criticisms.

\section{The low odds ratio: GWAS to blame?}

A frequent complaint is that GWAS results mean little in clinical situations due to the small effect of variants on disease risk and their relatively small contribution to common disease etiology. This claim might be partially correct. In the first place, "significantly associated" does not mean that the association identified by the study is significant in biology, medicine, or actual life. For example, a study reported the identification of an SNP that is associated with height, but its estimated additive effect is only $0.44 \mathrm{~cm}$ on height (Sanna et al., 2008). The majority of disease susceptibility loci identified by GWAS in different cancers have low effect size (per allele estimated effect size of 1.1-1.3) (Chung et al., 2010).

In such situations we can't just judge this gene is significant in the pathogenesis of PCa. But we should also take caution that in some situations, low odds ratio may be not due to the GWAS itself. In interpretation of these low odds ratio SNPs, we should firstly concentrate on the specific study character. For instance, using pooled controls of general populations as controls must decrease the ORs of the susceptibility alleles because of contamination of the potential patients in the controls. Secondly, ambiguous definition of populations, like "White Americans" or "Europeans", would compromise the result by admixture of different ethnic subgroups. Thus, it is necessary to detect population stratification prior to association analysis (Ikegawa, 2012). Last but not least, as genetic variants happens in different process, single nucleus mutations alone is far more not enough to be a major factor in the onset of PCa. The real "bad" guy among these SNPs is often the changes related with further genetic changes in other level. If we can find and allocate these related changes and evaluate their impact in a global view, it'll be easier to enhance our evidence proving the impact of these SNPs. Some scientists explore the GWAS data in a "pathway" fashion. They believe taking these data as a more concrete and more associated way would help to solve the "low-OR" issue.

\section{The difficulty in functional interpretation: is our mapping correct?}

When a specific SNP is identified, further mapping is performed to identify which gene the SNP is located. Much too often, these SNPs are intronic (45\%) or intergenic (43\%) (Hindorff et al., 2009). This brings difficulties for interpretation of the role these SNPs played in PCa pathogenesis. But sometimes the interpretation of the "mapping" results is wrong. In many studies, authors just make a story for causality by relating to known genes near the marker SNPs, even if they are far apart on the genome (Ikegawa, 2012). In some situations, it is not proper to associate these genes with $\mathrm{PCa}$ and even conduct functional studies on these genes.

This is also not the GWAS to blame as well. Since the con- 
cept of the "gene" itself is changing, GWASs can be a starting point to find unrevealed gene in the genome. There are new periods on the chromosome that used to be considered not as a gene but are recognized as a gene now. The "intergenic" loci may be located between two genes but they also might locate on the region of an unidentified gene. For example, the gene DVWA was reported to be associated with osteoarthritis. However, DVWA was first considered to be a gene desert in the time of the discovery of this SNP (Miyamoto et al., 2008). This means the assigning functional features of susceptibility SNPs and the understanding of "gene" should be a mutually beneficial process.

Even if we confirmed that one locus is not located in the potential gene area but in intergenic regions of the genome, its functional study may still meet unprecedented difficulties since functional features of these regions are still poorly defined. Recently, it was demonstrated that SNPs could be called from short sequenced tags acquired from Illumina sequencing during ChIP-Seq (Mikkelsen et al., 2007). It would be highly informative to know if TF (transcription factors) binding sites or chromatin-marked regulatory elements contain SNPs which might be used to determine regulatory SNPs (Hawkins et al., 2010). The SNP rs6983267 on 8q24, which has been identified as a PCa susceptibility locus, has been showed to have a functional role in colorectal cancer. As mentioned above, this SNP may have its impact on cancer risk through its nearest gene, c-MYC. In a functional study, researchers further show that the beta-catenin-TCF4 transcription factor complex binds preferentially to the cancer risk-associated allele in colon cancer cells (Wright et al., 2010). Researchers demonstrated that this SNP has enhancer-related histone marks and can form a 335-kb chromatin loop to interact with the c-MYC promoter (Wright et al., 2010).

The overwhelming information: what should we do with it?

The developing of GWAS offers scientists the greatest amount of information than ever before. Compared with the low throughput technologies before, the next generation sequencing technology made it possible to gain substantially larger amount of information within a short time interval. Nevertheless, the utilization of such information is still not as fully as we hoped.

Since single-allele association results published in many GWAS studies represent only the tip of the iceberg for the information that can be extracted from these datasets. Some researchers argue that analysis of the GWAS database in a pathway-based strategy would be beneficial to identify real meaningful alleles (Yaspan and Veatch, 2011). Factors such as locus heterogeneity, epistasis, and multiple genes conferring small effects contribute to the complexity of the genetic models underlying phenotype expression. Organizing individual SNPs into biologically meaningful groups would absolutely help to reveal the effects of minor perturbations to genes and pathways is desirable.

As mentioned, an online downloadable catalog of GWAS and SNP-trait associations has been established (http://www. genome.gov/gwastudies) and will surely facilitate a multifaceted analysis of published GWAS results (Hindorff et al., 2009). The release of data from GWAS to the scientific community has been much appreciated by scientists and some funding agencies now requiring data release. The US National Center for Biotechnology Information has developed the Database of Genotype and Phenotype to deposit and to offer access to GWAS information (http://www.ncbi.nlm.nih.gov/gap?Db=gap). This facilitates the construction of well-characterized phenotype and genotype data throughout populations. Further metaanalysis of published GWAS is an effective and cost-saving method to further combine the effectiveness of performed research. A meta-analysis of GWAS and replication studies indicated that 31 SNPs are correlated with PCa susceptibility (Liu et al., 2011). Combining all of the original data according to participants' ethnic origin, associations of some SNPs were evident in different ethnic groups. In other words, these "undiscovered changes" might be further explored by this economically beneficial method. Another meta-analysis identified prostate cancer susceptibility loci associated with aggressive and non-aggressive disease. As discussed above, few variants identified by GWAS have been shown to be associated with aggressive PCa, results of meta-analysis of GWAS have identified prostate cancer susceptibility loci associated with aggressive and non-aggressive disease (Amin Al Olama et al., 2013).

\section{GWAS: THE END OR THE BEGINNING?}

\section{Not even the end of the beginning}

After talking about all these common problems of GWAS, we may now draw the conclusion that the heat of GWAS is probably not going to be ending soon. Winston Churchill said at the victory against Nazi in North Africa, "This is not the end." If GWAS is just the beginning of the end, we are now not in the end of the beginning either. We need more GWAS to solve the deficiency of current researches.

\section{Not enough GWAS}

In summary, the evaluation of the GWAS in PCa at present stage should be there are not enough studies, not enough replications, even much less enough functional annotations. First, the discovered susceptibility loci seems to only have the ability to explain less than $30 \%$ of the heterogeneity of PCa pathogenesis (Amin Al Olama et al., 2013). How can we know that there are so many other loci undiscovered? There are a couple of reasons: current GWAS underpowered for low risk alleles; some known alleles have not shown up in GWAS. As the best currently available tool to detect PCa heredity, GWAS in PCa should be continued, of course maybe in the near future with the price of sequencing continued to decrease. Secondly, racial difference requires us to perform more GWAS in the 
"undetected" Asian and African population. Difference in races may help us explain the differences in epidemiology, pathogenesis, as well as different target therapies. The need for more replication studies is based on the fact that the number and quality of replication studies are far from good enough. To better standardize replication studies, NCI-NHGRI Working Group announced its guidelines to conduct replication studies (Studies et al., 2007). Further, gene-gene interaction studies call for much more participants than association studies do, which means gathering more samples in the replication period is a must. Replication is paramount in GWAS to confirm findings, and replication has been widely accepted as the gold standard to discern genuine genetic associations.

\section{What will future GWAS offer us?}

Our expectations for GWAS are far beyond risk prediction and current GWASs are mainly in the "bench period" without clinical application. Back to the two difficulties in PCa diagnosis and therapy mentioned in the beginning of this review, will GWAS help us dealing with these two difficulties in urgent need?

\section{GWAS findings for diagnostic biomarkers}

These discovered SNPs must be combined in a well-designed way to be translated into clinical practical biomarkers. Future GWAS will be able to find enough variants to form a genetic diagnosis score and a risk prediction score to establish a better prevention strategy. However, existing susceptibility SNPs have poor clinical performance in detecting $\mathrm{PCa}$ in a retrospective study in Chinese population (Zheng et al., 2012). The area under cure (AUC) of the receiver operating characteristic curve (ROC) is 0.604 less than that of PSA. The application of SNPs found in European ancestor into other population may be a possible explanation for this poor performance. Nevertheless, the findings from the REDUCE trial indicated the differences in population is not the only factor to be blamed (Kader et al., 2012). It is indicated that adding the genetic score to established clinical parameters would offer a modest improvement in PCa prediction. The genetic score based on 33 established SNPs may just improve better determining the need for repeat prostate biopsy. The absence of undiscovered variants may be another explanation yet the results may also inform us the need for the combination with other parameters for $\mathrm{PCa}$ risk prediction. At the meantime, performance of SNPs for PCa prognosis prediction in active surveillance cohort has been tested (Goh et al., 2013). Although the initial result is less than desirable, future investigations are guaranteed to further study.

\section{GWAS and molecular classification of PCa}

Although it is well recognized PCa is a set of heterogeneous diseases, there is not an established molecular classification system for $\mathrm{PCa}$ at the moment. Thus it is predictable that therapy for PCa which fight against the different enemies with the same strategy will actually have little effect on certain type of PCa. GWAS studies are completing a whole picture of the genetic heredity of $\mathrm{PCa}$ and in due course this genetic information from GWAS, along with somatic variants, would build the molecular classification criteria. Establishment of such system is the best and ultimate way towards personalized medicine.

\section{GWAS and pharmacogenomics}

It is expected that in the next decade, personalized medicine, or most recently termed precise medicine, will advance fast as one of the predominant strategic initiatives for researchers as well as pharmaceutical companies. To lay the foundation for personalized medicine, GWAS in pharmacogenomics can do a lot to help. As same as genetic diseases, it is hypothesized that variability in drug response, like efficacy or variability in target, is due to underlying variation in genetic information (Ritchie, 2012). Also, the effect size for many identified genetic associations for pharmacogenomic traits are much larger than those for common diseases (Ritchie, 2012). EGFR tyrosine kinase inhibitors (TKIs) in the treatment of lung cancer (Yi et al., 2009) and HER2-directed therapies in the treatment of HER2-positive early-stage breast cancer (Arteaga et al., 2012) are listed as successful examples for pharmacogenomic studies. Also, it has been proved that SNPs may influence the response and toxicity of Sunitinib in treatment of renal-cell carcinoma (GarciaDonas et al., 2011). However, current candidate SNPs studies are limited, thus many important SNPs may not be identified. If GWAS can identify SNPs that is associated with treatment response or tolerability, it would be a big step forward. For example, Methotrexate has been illustrated associated with Methotrexate clearance and gastrointestinal toxicity in paediatric acute lymphoblastic leukaemia patients (Trevino et al., 2009). In such respect, leading scientists give their opinion in pharmacogenomics study design (Wheeler et al., 2013), which future studies may take as a reference.

\section{Is GWAS fighting alone?}

The mostly discussed two weapons in the arsenal of genetic society may be microarrays and GWAS nowadays. Yet more fruitful results are expected to be yielded if we combine these two technologies. Researchers have performed an integrative pathway analysis of a GWAS dataset and a microarray gene expression dataset of PCa (Jia et al., 2012). Through this "cross-domain" strategy, more comprehensive pathways have been associated with PCa with more statistically significant results, such as "Fc gamma R-mediated phagocytosis", "regulation of actin cytoskeleton", and "Jak-STAT signaling pathway". However, the large amount of information and combining of different platforms may pose challenge for data access and processing, so it is quite for sure that strategies are still the hot topic to discuss in the future. What's more, to track the impact and functional annotations of established SNPs we need to combine information from different platforms. Epigenetics, transcriptomics, proteomics, and genomics all provide an insight to the genome function. Comprehensive analysis of information 
from chromatin immunoprecipitation followed by sequencing (ChIP-seq) and RNA sequencing (RNA-seq) are needed (Juran and Lazaridis, 2011). Also, it is predictable that in the future, more novel techniques will be established and facilitate our way of understanding prostate cancer pathogenesis.

\section{CONCLUSION}

In this review, we have discussed previous findings of GWAS in prostate cancer research and what it will offer us in the future. In the time of quite an abundance of GWAS, it is the time to pause and look back the way we've been through and choose the way we are going to explore. It is always important to keep in mind that GWAS must be translated into clinical use. With the advent of high-through technologies, whole genomic association techniques will cooperate with other techniques and a comprehensive system will be established. Although we are quite near the end of the beginning, post-GWAS studies are just taking off and future studies are needed extensively. Only in that day, GWAS information will be helpful in building a comprehensive care system intergraded with PCa prevention, diagnosis, molecular classification, and personalized therapy.

\section{ABBREVIATIONS}

CGEMS, Cancer Genetic Markers of Susceptibility; GWAS, genomewide association studies; MSMB, $\beta$-microseminoprotein; PCa, prostate cancer; PSA, prostate-specific antigen; SNPs, single-nucleotide polymorphisms

\section{COMPLIANCE WITH ETHICS GUIDELINES}

Rui Chen, Shancheng Ren and Yinghao Sun declare that they have no conflict of interest.

This article does not contain any studies with human or animal subjects performed by the any of the authors.

\section{REFERENCES}

Ahn, J., Berndt, S.I., Wacholder, S., Kraft, P., Kibel, A.S., Yeager, M., Albanes, D., Giovannucci, E., Stampfer, M.J., Virtamo, J., et al. (2008). Variation in KLK genes, prostate-specific antigen and risk of prostate cancer. Nat Genet 40, 1032-1034; author reply 10351036.

Ahn, J., Kibel, A.S., Park, J.Y., Rebbeck, T.R., Rennert, H., Stanford, J.L., Ostrander, E.A., Chanock, S., Wang, M.H., Mittal, R.D., et al. (2011). Prostate cancer predisposition loci and risk of metastatic disease and prostate cancer recurrence. Clin Cancer Res 17, 1075-1081.

Amin Al Olama, A., Kote-Jarai, Z., Schumacher, F.R., Wiklund, F., Berndt, S.I., Benlloch, S., Giles, G.G., Severi, G., Neal, D.E., Hamdy, F.C., et al. (2013). A meta-analysis of genome-wide association studies to identify prostate cancer susceptibility loci associated with aggressive and non-aggressive disease. Hum Mol Genet 22, 408-415.

Arteaga, C.L., Sliwkowski, M.X., Osborne, C.K., Perez, E.A., Puglisi, F., and Gianni, L. (2012). Treatment of HER2-positive breast cancer: current status and future perspectives. Nat Rev Clin Oncol 9,
$16-32$.

Bensen, J.T., Xu, Z., Smith, G.J., Mohler, J.L., Fontham, E.T., and Taylor, J.A. (2013). Genetic polymorphism and prostate cancer aggressiveness: a case-only study of 1,536 GWAS and candidate SNPs in African-Americans and European-Americans. Prostate 73, 11-22.

Berger, M.F., Lawrence, M.S., Demichelis, F., Drier, Y., Cibulskis, K., Sivachenko, A.Y., Sboner, A., Esgueva, R., Pflueger, D., Sougnez, C., et al. (2011). The genomic complexity of primary human prostate cancer. Nature 470, 214-220.

Chan, J.Y., Li, H., Singh, O., Mahajan, A., Ramasamy, S., Subramaniyan, K., Kanesvaran, R., Sim, H.G., Chong, T.W., Teo, Y.Y., et al. (2012). 8q24 and 17q Prostate cancer susceptibility loci in a multiethnic Asian cohort(论). Urol Oncol. (In press).

Chang, B.L., Cramer, S.D., Wiklund, F., Isaacs, S.D., Stevens, V.L., Sun, J., Smith, S., Pruett, K., Romero, L.M., Wiley, K.E., et al. (2009). Fine mapping association study and functional analysis implicate a SNP in MSMB at 10q11 as a causal variant for prostate cancer risk. Hum Mol Genet 18, 1368-1375.

Chang, B.L., Spangler, E., Gallagher, S., Haiman, C.A., Henderson, B., Isaacs, W., Benford, M.L., Kidd, L.R., Cooney, K., Strom, S., et al. (2011). Validation of genome-wide prostate cancer associations in men of African descent. Cancer Epidemiol Biomarkers Prev 20, 23-32.

Cheng, I., Chen, G.K., Nakagawa, H., He, J., Wan, P., Laurie, C.C., Shen, J., Sheng, X., Pooler, L.C., Crenshaw, A.T., et al. (2012). Evaluating genetic risk for prostate cancer among Japanese and Latinos. Cancer Epidemiol Biomarkers Prev 21, 2048-2058.

Chung, C.C., Magalhaes, W.C., Gonzalez-Bosquet, J., and Chanock, S.J. (2010). Genome-wide association studies in cancer--current and future directions. Carcinogenesis 31, 111-120.

Eeles, R.A., Kote-Jarai, Z., Al Olama, A.A., Giles, G.G., Guy, M., Severi, G., Muir, K., Hopper, J.L., Henderson, B.E., Haiman, C.A., et al. (2009). Identification of seven new prostate cancer susceptibility loci through a genome-wide association study. Nat Genet 41, 1116-1121.

Eeles, R.A., Kote-Jarai, Z., Giles, G.G., Olama, A.A., Guy, M., Jugurnauth, S.K., Mulholland, S., Leongamornlert, D.A., Edwards, S.M., Morrison, J., et al. (2008). Multiple newly identified loci associated with prostate cancer susceptibility. Nat Genet 40, 316-321.

Elston, R.C., Lin, D., and Zheng, G. (2007). Multistage sampling for genetic studies. Annu Rev Genomics Hum Genet 8, 327-342.

FitzGerald, L.M., Kwon, E.M., Conomos, M.P., Kolb, S., Holt, S.K., Levine, D., Feng, Z., Ostrander, E.A., and Stanford, J.L. (2011). Genome-wide association study identifies a genetic variant associated with risk for more aggressive prostate cancer. Cancer Epidemiol Biomarkers Prev 20, 1196-1203.

FitzGerald, L.M., Zhang, X., Kolb, S., Kwon, E.M., Liew, Y.C., HurtadoColl, A., Knudsen, B.S., Ostrander, E.A., and Stanford, J.L. (2013). Investigation of the relationship between prostate cancer and MSMB and NCOA4 genetic variants and protein expression. Hum Mutat 34, 149-156.

Freedman, M.L., Monteiro, A.N., Gayther, S.A., Coetzee, G.A., Risch, A., Plass, C., Casey, G., De Biasi, M., Carlson, C., Duggan, D., et al. (2011). Principles for the post-GWAS functional characterization of cancer risk loci. Nat Genet 43, 513-518.

Garcia-Donas, J., Esteban, E., Leandro-Garcia, L.J., Castellano, D.E., 
del Alba, A.G., Climent, M.A., Arranz, J.A., Gallardo, E., Puente, J., Bellmunt, J., et al. (2011). Single nucleotide polymorphism associations with response and toxic effects in patients with advanced renal-cell carcinoma treated with first-line sunitinib: a multicentre, observational, prospective study. Lancet Oncol 12, 1143-1150.

Goh, C.L., Saunders, E.J., Leongamornlert, D.A., Tymrakiewicz, M., Thomas, K., Selvadurai, E.D., Woode-Amissah, R., Dadaev, T., Mahmud, N., Castro, E., et al. (2013). Clinical implications of family history of prostate cancer and genetic risk single nucleotide polymorphism (SNP) profiles in an active surveillance cohort. BJU Int. (In Press).

Gudmundsson, J., Sulem, P., Rafnar, T., Bergthorsson, J.T., Manolescu, A., Gudbjartsson, D., Agnarsson, B.A., Sigurdsson, A., Benediktsdottir, K.R., Blondal, T., et al. (2008). Common sequence variants on $2 \mathrm{p} 15$ and $\mathrm{Xp} 11.22$ confer susceptibility to prostate cancer. Nat Genet 40, 281-283.

Gudmundsson, J., Sulem, P., Steinthorsdottir, V., Bergthorsson, J.T., Thorleifsson, G., Manolescu, A., Rafnar, T., Gudbjartsson, D., Agnarsson, B.A., Baker, A., et al. (2007). Two variants on chromosome 17 confer prostate cancer risk, and the one in TCF2 protects against type 2 diabetes. Nat Genet 39, 977-983.

Haiman, C.A., Chen, G.K., Blot, W.J., Strom, S.S., Berndt, S.I., Kittles, R.A., Rybicki, B.A., Isaacs, W.B., Ingles, S.A., Stanford, J.L., et al. (2011). Characterizing genetic risk at known prostate cancer susceptibility loci in African Americans. PLoS Genet 7, e1001387.

Hawkins, R.D., Hon, G.C., and Ren, B. (2010). Next-generation genomics: an integrative approach. Nat Rev Genet 11, 476-486.

Hindorff, L.A., Sethupathy, P., Junkins, H.A., Ramos, E.M., Mehta, J.P., Collins, F.S., and Manolio, T.A. (2009). Potential etiologic and functional implications of genome-wide association loci for human diseases and traits. Proc Natl Acad Sci U S A 106, 9362-9367.

Hirschhorn, J.N., and Daly, M.J. (2005). Genome-wide association studies for common diseases and complex traits. Nat Rev Genet 6 , 95-108.

Hooker, S., Hernandez, W., Chen, H., Robbins, C., Torres, J.B., Ahaghotu, C., Carpten, J., and Kittles, R.A. (2010). Replication of prostate cancer risk loci on 8q24, 11q13, 17q12, 19q33, and Xp11 in African Americans. Prostate 70, 270-275.

Ikegawa, S. (2012). A short history of the genome-wide association study: where we were and where we are going. Genomics Inform 10, 220-225.

Ishak, M.B., and Giri, V.N. (2011). A systematic review of replication studies of prostate cancer susceptibility genetic variants in high-risk men originally identified from genome-wide association studies. Cancer Epidemiol Biomarkers Prev 20, 1599-1610.

Jia, P., Liu, Y., and Zhao, Z. (2012). Integrative pathway analysis of genome-wide association studies and gene expression data in prostate cancer. BMC Syst Biol 6 Suppl 3, S13.

Juran, B.D., and Lazaridis, K.N. (2011). Genomics in the post-GWAS era. Semin Liver Dis 31, 215-222.

Kader, A.K., Sun, J., Reck, B.H., Newcombe, P.J., Kim, S.T., Hsu, F.C., D’Agostino, R.B., Jr., Tao, S., Zhang, Z., Turner, A.R., et al. (2012). Potential impact of adding genetic markers to clinical parameters in predicting prostate biopsy outcomes in men following an initial negative biopsy: findings from the REDUCE trial. Eur Urol 62, 953-961.

Kerns, S.L., Stock, R., Stone, N., Buckstein, M., Shao, Y., Campbell,
C., Rath, L., De Ruysscher, D., Lammering, G., Hixson, R., et al. (2013). A 2-stage genome-wide association study to identify single nucleotide polymorphisms associated with development of erectile dysfunction following radiation therapy for prostate cancer. Int J Radiat Oncol Biol Phys 85, e21-28.

Kim, H.J., Bae, J.S., Lee, J., Chang, I.H., Kim, K.D., Shin, H.D., Han, J.H., Lee, S.Y., Kim, W., and Myung, S.C. (2011). HNF1B polymorphism associated with development of prostate cancer in Korean patients. Urology 78, 969. e1-6.

Konety, B.R., Bird, V.Y., Deorah, S., and Dahmoush, L. (2005). Comparison of the incidence of latent prostate cancer detected at autopsy before and after the prostate specific antigen era. J Urol 174, 1785-1788; discussion 1788.

Lai, J., Kedda, M.A., Hinze, K., Smith, R.L., Yaxley, J., Spurdle, A.B., Morris, C.P., Harris, J., and Clements, J.A. (2007). PSA/KLK3 AREI promoter polymorphism alters androgen receptor binding and is associated with prostate cancer susceptibility. Carcinogenesis 28 , 1032-1039.

Lichtenstein, P., Holm, N.V., Verkasalo, P.K., Iliadou, A., Kaprio, J., Koskenvuo, M., Pukkala, E., Skytthe, A., and Hemminki, K. (2000). Environmental and heritable factors in the causation of cancer-analyses of cohorts of twins from Sweden, Denmark, and Finland. N Engl J Med 343, 78-85.

Liu, H., Wang, B., and Han, C. (2011). Meta-analysis of genome-wide and replication association studies on prostate cancer. Prostate 71 , 209-224.

Lou, H., Li, H., Yeager, M., Im, K., Gold, B., Schneider, T.D., Fraumeni, J.F., Jr., Chanock, S.J., Anderson, S.K., and Dean, M. (2012). Promoter variants in the MSMB gene associated with prostate cancer regulate MSMB/NCOA4 fusion transcripts. Hum Genet 131, 14531466.

Mehra, R., Han, B., Tomlins, S.A., Wang, L., Menon, A., Wasco, M.J., Shen, R., Montie, J.E., Chinnaiyan, A.M., and Shah, R.B. (2007). Heterogeneity of TMPRSS2 gene rearrangements in multifocal prostate adenocarcinoma: molecular evidence for an independent group of diseases. Cancer Res 67, 7991-7995.

Mikkelsen, T.S., Ku, M., Jaffe, D.B., Issac, B., Lieberman, E., Giannoukos, G., Alvarez, P., Brockman, W., Kim, T.K., Koche, R.P., et al. (2007). Genome-wide maps of chromatin state in pluripotent and lineage-committed cells. Nature 448, 553-560.

Miyamoto, Y., Shi, D., Nakajima, M., Ozaki, K., Sudo, A., Kotani, A., Uchida, A., Tanaka, T., Fukui, N., Tsunoda, T., et al. (2008). Common variants in DVWA on chromosome 3p24.3 are associated with susceptibility to knee osteoarthritis. Nat Genet 40, 994-998.

Nam, R.K., Zhang, W., Siminovitch, K., Shlien, A., Kattan, M.W., Klotz, L.H., Trachtenberg, J., Lu, Y., Zhang, J., Yu, C., et al. (2011). New variants at $10 q 26$ and $15 q 21$ are associated with aggressive prostate cancer in a genome-wide association study from a prostate biopsy screening cohort. Cancer Biol Ther 12, 997-1004.

Panagopoulos, I., Moller, E., Collin, A., and Mertens, F. (2008). The POU5F1P1 pseudogene encodes a putative protein similar to POU5F1 isoform 1. Oncol Rep 20, 1029-1033.

Prokunina-Olsson, L., Fu, Y.P., Tang, W., Jacobs, K.B., Hayes, R.B., Kraft, P., Berndt, S.I., Wacholder, S., Yu, K., Hutchinson, A., et al. (2010). Refining the prostate cancer genetic association within the JAZF1 gene on chromosome 7p15.2. Cancer Epidemiol Biomarkers Prev 19, 1349-1355. 
Ritchie, M.D. (2012). The success of pharmacogenomics in moving genetic association studies from bench to bedside: study design and implementation of precision medicine in the post-GWAS era. Hum Genet 131, 1615-1626.

Sanna, S., Jackson, A.U., Nagaraja, R., Willer, C.J., Chen, W.M., Bonnycastle, L.L., Shen, H., Timpson, N., Lettre, G., Usala, G., et al. (2008). Common variants in the GDF5-UQCC region are associated with variation in human height. Nat Genet 40, 198-203.

Schumacher, F.R., Berndt, S.I., Siddiq, A., Jacobs, K.B., Wang, Z., Lindstrom, S., Stevens, V.L., Chen, C., Mondul, A.M., Travis, R.C., et al. (2011). Genome-wide association study identifies new prostate cancer susceptibility loci. Hum Mol Genet 20, 3867-3875.

Seng, K.C., and Seng, C.K. (2008). The success of the genome-wide association approach: a brief story of a long struggle. Eur J Hum Genet 16, 554-564.

Severi, G., Hayes, V.M., Neufing, P., Padilla, E.J., Tilley, W.D., Eggleton, S.A., Morris, H.A., English, D.R., Southey, M.C., Hopper, J.L., et al. (2006). Variants in the prostate-specific antigen (PSA) gene and prostate cancer risk, survival, and circulating PSA. Cancer Epidemiol Biomarkers Prev 15, 1142-1147.

Siegel, R., Naishadham, D., and Jemal, A. (2013). Cancer statistics, 2013. CA Cancer J Clin 63, 11-30.

Sotelo, J., Esposito, D., Duhagon, M.A., Banfield, K., Mehalko, J., Liao, H., Stephens, R.M., Harris, T.J., Munroe, D.J., and Wu, X. (2010). Long-range enhancers on 8q24 regulate c-Myc. Proc Natl Acad Sci U S A 107, 3001-3005.

Stadler, Z.K., Thom, P., Robson, M.E., Weitzel, J.N., Kauff, N.D., Hurley, K.E., Devlin, V., Gold, B., Klein, R.J., and Offit, K. (2010). Genome-wide association studies of cancer. J Clin Oncol 28, 4255-4267.

Stevens, V.L., Ahn, J., Sun, J., Jacobs, E.J., Moore, S.C., Patel, A.V., Berndt, S.I., Albanes, D., and Hayes, R.B. (2010). HNF1B and JAZF1 genes, diabetes, and prostate cancer risk. Prostate 70 , 601-607.

Studies, N.-N.W.G.o.R.i.A., Chanock, S.J., Manolio, T., Boehnke, M., Boerwinkle, E., Hunter, D.J., Thomas, G., Hirschhorn, J.N., Abecasis, G., Altshuler, D., et al. (2007). Replicating genotype-phenotype associations. Nature 447, 655-660.

Takata, R., Akamatsu, S., Kubo, M., Takahashi, A., Hosono, N., Kawaguchi, T., Tsunoda, T., Inazawa, J., Kamatani, N., Ogawa, O., et al. (2010). Genome-wide association study identifies five new susceptibility loci for prostate cancer in the Japanese population. Nat Genet 42, 751-754.

Thomas, G., Jacobs, K.B., Yeager, M., Kraft, P., Wacholder, S., Orr, N., Yu, K., Chatterjee, N., Welch, R., Hutchinson, A., et al. (2008). Multiple loci identified in a genome-wide association study of prostate cancer. Nat Genet 40, 310-315.

Trevino, L.R., Shimasaki, N., Yang, W., Panetta, J.C., Cheng, C., Pei, D., Chan, D., Sparreboom, A., Giacomini, K.M., Pui, C.H., et al. (2009). Germline genetic variation in an organic anion transporter polypeptide associated with methotrexate pharmacokinetics and clinical effects. J Clin Oncol 27, 5972-5978.

Vickers, A., Cronin, A., Roobol, M., Savage, C., Peltola, M., Petters- son, K., Scardino, P.T., Schroder, F., and Lilja, H. (2010). Reducing unnecessary biopsy during prostate cancer screening using a four-kallikrein panel: an independent replication. J Clin Oncol 28, 2493-2498.

Wang, M., Liu, F., Hsing, A.W., Wang, X., Shao, Q., Qi, J., Ye, Y., Wang, Z., Chen, H., Gao, X., et al. (2012). Replication and cumulative effects of GWAS-identified genetic variations for prostate cancer in Asians: a case-control study in the ChinaPCa consortium. Carcinogenesis 33, 356-360.

Wasserman, N.F., Aneas, I., and Nobrega, M.A. (2010). An 8q24 gene desert variant associated with prostate cancer risk confers differential in vivo activity to a MYC enhancer. Genome Res 20, 1191-1197.

Wheeler, H.E., Maitland, M.L., Dolan, M.E., Cox, N.J., and Ratain, M.J. (2013). Cancer pharmacogenomics: strategies and challenges. Nat Rev Genet 14, 23-34.

Wright, J.B., Brown, S.J., and Cole, M.D. (2010). Upregulation of CMYC in cis through a large chromatin loop linked to a cancer riskassociated single-nucleotide polymorphism in colorectal cancer cells. Mol Cell Biol 30, 1411-1420.

Xu, J., Mo, Z., Ye, D., Wang, M., Liu, F., Jin, G., Xu, C., Wang, X., Shao, Q., Chen, Z., et al. (2012). Genome-wide association study in Chinese men identifies two new prostate cancer risk loci at 9q31.2 and 19q13.4. Nat Genet 44, 1231-1235.

Xu, J., Zheng, S.L., Isaacs, S.D., Wiley, K.E., Wiklund, F., Sun, J., Kader, A.K., Li, G., Purcell, L.D., Kim, S.T., et al. (2010). Inherited genetic variant predisposes to aggressive but not indolent prostate cancer. Proc Natl Acad Sci U S A 107, 2136-2140.

Yaspan, B.L., and Veatch, O.J. (2011). Strategies for pathway analysis from GWAS data. Curr Protoc Hum Genet. Chapter 1, Unit1.20.

Yeager, M., Chatterjee, N., Ciampa, J., Jacobs, K.B., Gonzalez-Bosquet, J., Hayes, R.B., Kraft, P., Wacholder, S., Orr, N., Berndt, S., et al. (2009). Identification of a new prostate cancer susceptibility locus on chromosome 8q24. Nat Genet 41, 1055-1057.

Yeager, M., Orr, N., Hayes, R.B., Jacobs, K.B., Kraft, P., Wacholder, S., Minichiello, M.J., Fearnhead, P., Yu, K., Chatterjee, N., et al. (2007). Genome-wide association study of prostate cancer identifies a second risk locus at 8q24. Nat Genet 39, 645-649.

Yi, H.G., Kim, H.J., Kim, Y.J., Han, S.W., Oh, D.Y., Lee, S.H., Kim, D.W., Im, S.A., Kim, T.Y., Kim, C.S., et al. (2009). Epidermal growth factor receptor (EGFR) tyrosine kinase inhibitors (TKIs) are effective for leptomeningeal metastasis from non-small cell lung cancer patients with sensitive EGFR mutation or other predictive factors of good response for EGFR TKI. Lung Cancer 65, 80-84.

Zeggini, E., Scott, L.J., Saxena, R., Voight, B.F., Marchini, J.L., Hu, T., de Bakker, P.I., Abecasis, G.R., Almgren, P., Andersen, G., et al. (2008). Meta-analysis of genome-wide association data and largescale replication identifies additional susceptibility loci for type 2 diabetes. Nat Genet 40, 638-645.

Zheng, J., Liu, F., Lin, X., Wang, X., Ding, Q., Jiang, H., Chen, H., Lu, D., Jin, G., Hsing, A.W., et al. (2012). Predictive performance of prostate cancer risk in Chinese men using 33 reported prostate cancer risk-associated SNPs. Prostate 72, 577-583. 